\title{
Editorial
}

\section{Using guidelines for coronary revascularisation: how many are needed and are they any good?}

Gone are the days when guidelines could be ignored as cookbook medicine, the overweening influence of purchasers, or a simplistic view of the world with little value in practical patient management. Most would now accept the broad principle that it is a part of good clinical medicine to assess practice against standards linked explicitly to scientific evidence. In this issue Peters and Bredee ${ }^{1}$ discuss new guidelines from the Netherlands on coronary revascularisation and make a bold proposal for their use in practice. What sense are we to make of this among the plethora of guidelines and quality of care studies?

\section{Guidelines: proliferation or unification?}

Guidelines, scarce 10 years ago, now abound; most areas of suspected and proved coronary artery disease are now covered by one or more guideline. In the US, aspects of the invasive management of coronary disease are dealt with in procedure specific guidelines (on exercise electrocardiography, coronary angiography, angioplasty, stents, coronary artery bypass grafts) as well as guidelines for separate clinical presentations (chronic stable angina, unstable angina, acute myocardial infarction) issued by the American College of Cardiology/American Heart Association (www.acc.org/clinical/guidelines/index.html) or Agency for Health Care Policy and Research. It is possible therefore that the care of a single cardiac patient may come under the remit of half a dozen different guidelines from the same organisation. The European Society of Cardiology (www.escardio.org) has issued guidelines on the management of chronic stable angina and myocardial infarction, as has the British Cardiac Society (www.bcs.com). Neither organisation has published guidelines specifically on the invasive management of coronary disease.

As Peters and Bredee describe, national health policy, rather than professional bodies, may dictate the need for national guidelines. The UK may follow the Netherlands, with the National Service Framework for Coronary Heart Disease ${ }^{2}$ issuing standards of "predetermined criteria" by which patients should be selected for revascularisation. The newly established National Institute for Clinical Excellence will have responsibility for developing such criteria and practice will be inspected by the Commission for Health Improvement.

To what extent does each country need its own guidelines? Can each country claim to have unique combinations of patient characteristics; rates of referral, investigation and treatment; health care systems and funding; and professional organisations? Or is good clinical medicine the same in Haarlem, Netherlands as in Harlem, USA? After all, the evidence that informs guidelines is essentially unitary (even if most of it comes from the US) and the considerable exercise of systematically reviewing ${ }^{3}$ this evidence or in grading the strength of evidence for each recommendation ${ }^{4}$ should not be duplicated without good reason.

The answers to these questions depend on the clinical decisions covered by the guidelines. In the management of unselected patients presenting with chest pain, the argument for materially different guideline recommendations in specific countries is strong. In such situations many countries may legitimately argue that they are special, and service availability, health economic, and other factors are rightly allowed influence. However, by the time the patient has undergone coronary angiography and reached the point of being considered for revascularisation, the argument for major inter-nation differences in recommendations for good clinical practice is less strong. Might one core international guideline suffice, possibly with minor modification to local circumstances? After all how many different recommendations for the management of triple vessel disease, once demonstrated, can there be?

\section{Guidelines: any good?}

If guidelines abound, their use in routine clinical practice does not. To date, guidelines have been used more in ad hoc audits and quality of care studies than in everyday practice. What is to say if a guideline is any good? The acid test is not just whether it influences the processes of medical care ${ }^{5}$ but whether it helps deliver better clinical outcomes to patients. Two related aspects of the guidelines discussed by Peters and Bredee are different from previous guidelines and might conceivably improve the quality of care. First, they explicitly force consideration of the question "should this patient undergo revascularisation?" This is one of the first attempts to address the generic question of coronary revascularisation, rather than the specific and separate questions of recommendations for angioplasty and bypass surgery. This novel approach may offer the opportunity to see the "wood for the trees" in a way that separate guidelines devoted to angioplasty or bypass surgery may not.

Second, Peters and Bredee make a specific proposal for the obligatory use of the guidelines in day to day clinical decision making - a proposal that is radically different from current practice in many countries. According to Peters and Bredee, " . . it is necessary to discuss all patients who are considered for either elective or emergency revascularisation during a multi-disciplinary meeting [where] at least one interventional cardiologist and one cardiac surgeon should be present." This "heart team" will then, according to their published guidelines, decide the necessity and modality of subsequent treatment. If revascularisation is recommended, consideration is given to benefits (prognostic or symptomatic), risks (of intervention), and urgency (with estimated waiting time). A written report of the decision and considerations is then sent to the referring cardiologist.

Guidelines based review of all revascularisation decisions by both cardiologist and surgeon offers potential advantages. In many countries cardiologists "refer to themselves" patients for angioplasty and act as gatekeepers to cardiac surgery. If cardiologists had systematic preferences for their own procedure, a "heart team" might help improve the appropriateness of management by removing their gatekeeping role. However a recent UK expert panel rating the appropriateness of angioplasty and bypass surgery did not find any evidence that cardiologists rated 
angioplasty more appropriate than surgeons, nor that surgeons rated bypass more appropriate than cardiologists. ${ }^{6}$ The "heart team", by reaching consensus, might reduce ubiquitous practice variations. It would help reduce the error inherent in angiographic interpretation and disagreements on subsequent patient management. ${ }^{7}$ There might also be an opportunity to include explicitly the wishes of individual patients. Informed patient preference for revascularisation modality is essential as, for most patients being revascularised, neither bypass surgery nor angioplasty is superior across all outcomes at all periods of follow up, and people with a given level of functional impairment from angina place different values on symptomatic relief. ${ }^{8}$

However, potential advantages are not necessarily real advantages - they require empirical demonstration. Using guidelines as an ongoing aid to deciding whether and how to revascularise - rather than for one off, periodic auditconstitutes a health technology in its own right. Furthermore this is a technology with its own potential adverse consequences; there is a significant opportunity cost in involving surgeon and cardiologist in reviewing all patients for revascularisation. To address the question of whether these guidelines and the "heart team" approach are any better than usual decision making, comparative studies of clinical outcome are required. Prospective studies would need to measure clinical outcomes assessed by doctors and patients as well as cost and process measures (overall and procedure specific revascularisation rates and their variation between referring cardiologists and institutions; error in angiographic reporting; patient choice; waiting times).

Over the past decade, guidelines have been introduced that cover most aspects of the management of suspected and proved coronary artery disease. These guidelines are important sources of standards and their use may improve the process of medical care. ${ }^{5}$ However, guidelines are merely tools, not an inevitable feature of the practice of clinical medicine. If over the next decade such guidelines do not demonstrate their effectiveness (impact on clinical outcomes) alongside other health technologies, their days may be numbered.

HARRY HEMINGWAY

Department of Research and Development,

Kensington $\mathcal{E}$ Chelsea and Westminster Health Authority,

50 Eastbourne Terrace, London W2 6LX, UK

Department of Epidemiology and Public Health,

SHRILLA BANERJEE

University College London Medical School,

1-19 Torrington Place, London WC1E 6BT, UK

ADAM TIMMIS

Royal London and St Bartholomew's NHS Trust,

London EC1A 7BE, UK

1 Peters RJG, Bredee JJ. Indications for coronary revascularisations: a guideline for the Netherlands. Heart 2000;83:3-4.

2 Department of Health. National service framework for coronary heart disease. Health Service Circular 1998;218

3 Sculpher MJ, Petticrew M, Kelland JL, et al. Resource allocation for chronic stable angina: a systematic review of effectiveness, costs and costeffectiveness of alternative interventions. Health Technol Assess 1998;2:1176.

4 Scanlon PJ, Faxon DP, Audet AM, et al. ACC/AHA guidelines for coronary angiography. A report of the American College of Cardiology/American Heart Association Task Force on practice guidelines (Committee on Coronary Angiography). Developed in collaboration with the Society for Cardiac Angiography and Interventions. F Am Coll Cardiol 1999;33:1756-824.

5 Grimshaw JM, Russell IT. Effect of clinical guidelines on medical practice: a systematic review of rigorous evaluations [see comments]. Lancet 1993;342:1317-22

6 Hemingway H, Crook AM, Dawson R, et al. Rating the appropriateness of coronary angiography, coronary angioplasty and coronary artery bypass grafting: the ACRE study. F Public Health Med 1999;21:421-9.

7 Banerjee S, Crook AM, Dawson R, et al. The magnitude and consequences of error in coronary angiography interpretation: the ACRE study. $A m \mathcal{F}$ Cardiol. [In press.]

8 Nease RF Jr, Kneeland T, O'Connor GT, et al. Variation in patient utilities for outcomes of the management of chronic stable angina. Implications for clinical practice guidelines. Ischemic Heart Disease Patient Outcomes Research Team [see comments] [published erratum appears in $\mathscr{F} A M A$ 1995;274:612]. FAMA 1995;273:1185-90. 